\title{
On Controlling the Extent of Robust Solution in Uncertain Environment in Multiobjective Optimization
}

\author{
Tohid Erfani* and Sergei V.Utyuzhnikov ${ }^{\dagger}$ \\ The University of Manchester, School of Mechanical, Aerospace and Civil Engineering, \\ Manchester, M60 1QD, Great Britain
}

\begin{abstract}
In design and optimization problems, a solution which is stable enough in its variability in presence of the perturbation of the parameters/variables of the model is called robust. In engineering design optimization, the designer may sacrifice the optimal solution for the robust one in order to set a stable system design. Although in literature there are methods for capturing such solution, they do not provide the designer with a direct and systematic control over the level of the required robustness. In this paper a new robust design methodology is introduced in the context of the multiobjective optimization problem. The proposed approach not only is able to capture the robust design but also can introduce a manipulation for controlling the extent of the design's robustness based on the designer preferences while dealing with the fuzzy and interval values uncertainty. The presented method is different form its other counterparts as it does not average the vicinity of the current design nor change/add any new constraint to keep the feasibility of the design despite of variation. On the contrary, only a special tunable objective function is constructed to incorporate the preference of the designer on the extent of the demanded robustness efficiently. The benefits of the new approach are discussed and, to show its effectiveness, it is tested on a well known engineering design problem and the results are presented. The proposed approach is of immediate use for any engineering design, where the designer would like to study a set of robust solution with different levels and to choose the most appropriate ones amongst.
\end{abstract}

\section{Introduction}

TRIATION in operation caused by user and uncertainty about the model parameters make an engineering design setting to be changed from time to time. This sensitivity makes the designer to be less reluctant on finding only the optimal solution but rather try to seek for the most stable one. Such solution is called robust and is the matter of research in literature.

The work by Taguchi ${ }^{1,2}$ is considered amongst the first efforts in finding the robust design. In particular, they introduced a method in order to consider the effects of the model uncontrollable parameters and to minimize them during the design. The so called Taguchi's loss function ${ }^{3}$ is the proposed methodology in order to make design more tolerable by the model variations. Based on this concept other researchers $\left(^{4-8}\right)$, used optimization in order to introduce a more efficient methodology for minimizing the variation. By doing so, the less sensitive design is found by means of nonlinear optimization. They proposed a robust design formulation with Taguchi's loss function as an objective function subject to the model constraints. Implementing this, the constant and variable sensitivity from controllable and uncontrollable parameters are minimized respectively using nonlinear programming.

In single objective optimization, Parkinson ${ }^{9}$ works is considered as efforts for finding the robust solution by studying two main issues; feasibility of the design and the control of transmitted variation. In this work, the size of the feasible space is reduced in order to find the robust solution unaffected by the model variation. In addition of keeping the design feasible, the sensitivity of the design is minimized during the optimization process. This work is done by a nonlinear optimal design formulation. Analysis of tolerance ${ }^{10}$ including

*Post Graduate Researcher, AIAA Student Member, Email: tohid.erfani@postgrad.manchester.ac.uk

†Senior Research Fellow, School of MACE 
both worst case and statistical analysis are implemented to account for the transmitted variation of the parameters into the design functions.

$\mathrm{Su}$ and Renaud, ${ }^{11}$ on the other hand, developed another kind of robust design approach in single objective optimization. They introduced sensitivity-based and experimental-based robust design optimization to find the less sensitive optimum solution. The essence of these two approaches is to study the vicinity of current design and hence find the solution which is less sensitive to fluctuations of parameters and design variables.

In multiobjective optimization perspective, Messac and Yahaya ${ }^{12}$ develop a procedure in order to account for the flexible robust optimization. Using physical programming lexicon, ${ }^{13}$ a system with a minimal variation in the input variability and uncertainties is found while the feasibility of the design solution is guaranteed. They consider robust design with both prescribed and variable tolerances, in which the variable tolerance level is determined optimally in the design phase.

In another study and by extending an approach in single objective optimization, Deb and Gupta, also, developed a method to search for the robust solution in the multiobjective optimization. ${ }^{14}$ Two types of robust solutions are considered in different perspectives. The type one is to optimize the mean effective objective functions and to account for the extent of robustness. Type two is used by adding an additional constraint to the problem with a free parameter as its right hand side set by designer. Here again, the essence of the work is to minimize the feasible space in order to keep the design solution feasible during the optimization. As argued in Ref., ${ }^{14}$ depending on the sample population in the vicinity of each design, this method is computationally expensive as much as regular optimization methods.

In another effort, response surface methodology has been exploited by Chen et al, ${ }^{15}$ to substitute the original functions for the simpler ones. To account for the robust solution, then, they simultaneously optimize the mean of the performance and minimize the variation of the response formulated in a bi-objective optimization problem. Clearly, this provides a trade-off between the optimality and the robustness. However, the proposed approach is introduced in the context of single objective optimization.

Independent of the Taguchi work, idea of reducing the feasible space for feasibility robustness and the research on averaging a finite set of neighboring solution, we proposed a method not only to capture the robust design but also to control the extent of robustness based on the designer preferences in presence of uncertainty. In particular, we considered the fuzzy and interval value uncertainty of the parameters, which is common in engineering design, and extend a function as a measure for robustness for multiobjective optimization. It is shown that the proposed method is capable to capture different level of robust solution only based on introducing a new objective function. Interestingly, we do not add any new constraint or even change the existing ones.

Rest of the paper is organized as follows. Section II describes the robustness in optimization and includes all the definitions needed in the interim of the paper. The fuzzy and interval valued uncertainty in the model is handled in section III and the robust measure objective function is proposed within the same section. In section A, a new approach to find the desirable robust solution is discussed and its importance is stressed. The proposed approach is tested on an engineering designs in section IV accompanying with the numerical results. Concluding remarks are summarized in Section V.

\section{Handling uncertainty and robustness in optimization}

In engineering design optimization problems, one faces multi goals which objectives required to be set at their optimum level subject to some inevitable hard constraints. A generic multiobjective optimization problem takes the following form:

$$
\begin{aligned}
& \text { Min } \mathcal{F}=\left\{F_{1}(x), F_{2}(x), \ldots, F_{n}(x)\right\}, \\
& \text { subject to } x \in \mathcal{D}^{*} .
\end{aligned}
$$

As the solution to this problem is not unique, we seek a set of solutions called the Pareto. In the objective space, Pareto solutions forms the Pareto frontier, which gives the best trade-off solutions to the multiobjective optimisation problem (1). ${ }^{16}$

However, the design process is subject to some inevitable noise and uncertainties during the performance modeling. This will cause the model uncertainty. In this study, we consider the uncertainty of the parameters using fuzzy variables, though, it is possible to implement the same methodology for any kind of uncertainty 
within the model. Hence, the problem 1 changes to:

$$
\begin{aligned}
\text { Min } & \tilde{\mathcal{F}}=\left\{\tilde{F}_{1}(x), \tilde{F}_{2}(x), \ldots, \tilde{F}_{n}(x)\right\} \\
\text { subject to } & \widetilde{g_{i}(x)} \leq \tilde{b}_{i} \quad i=1 \ldots m \\
& x \in \mathcal{D},
\end{aligned}
$$

where tilde implies that the parameters of the model are not precisely known but are modeled using fuzzy variables.

To solve the problem 2 for its optimum values, the model should be converted into the deterministic formulation. For this purpose the nominal value of the parameters may be used. To do so, we substitute the fuzzy variables with their fuzzy possibilistic mean value. ${ }^{17}$ The problem, then, is deterministic and any multiobjective algorithm can be used to generate the set of Pareto optimal solution for it.

As stated earlier, variation in the model components turn the attention of the designer to ask for a more stable solutions than the optimal ones, though the optimal solution yields the better design. This leads to find the robust solution which is less sensitive in its variation in presence of the other model variability. Bearing this in mind, in the followings, we define a robust measure to account for robustness in multiobjective optimization.

\section{Robust measure construction}

To realize the robust solution, in literature, the optimal solution is shifted into the feasible space by making the design space smaller and accepting the degradation of the optimal value. ${ }^{9}$ This brings the need to change the constraints and find the transmitted variation for each of them, which is not timely efficient. Also, unless the source of variability is known, changing the right hand side of the problem is an ad-hoc process for maintaining the feasibility percentage. That is, there is not any control on or even understanding of the extent of the robustness of the solution, if the variability is interval value or purely the designer's specification about the system. Herein we address a new approach in searching for robust solution without changing the constraint rather introducing a function to be considered as a new objective function.

Based on the robustness concept, one may take a robust measure in it general formulation as follows: ${ }^{17}$

$$
\mathcal{R}_{\mathcal{F}}=\frac{1}{n m} \sum_{i=1}^{n} \sum_{j=1}^{m} \frac{\sigma F_{i}}{\sigma x_{j}}
$$

where $m$ and $n$ are the number of design components (parameters and variables, whichever is varied by designers' specification) and objective functions, respectively. $\sigma x_{j}$ denotes the variance of the (fuzzy or interval) parameter. To estimate the $\sigma F$ ( $i$ is omitted for simplicity), first order Taylor's series is implemented as follows:

$$
\sigma_{F}^{2}=\sum_{i=1}^{n}\left(\frac{\partial F}{\partial p_{i}}\right)^{2} \sigma_{p_{i}}^{2} .
$$

where $p$ account for the parameters of the model subject to variations. In (4), the designer states the variance of the parameters denoted as $\sigma_{p}$. For the sake of having the fuzzy or interval value parameters, the fuzzy variance can be used (Look at e.g. ${ }^{17}$ ).

However, one thing is overlooked and it is how to control the extent of the robustness.

\section{A. Controlling the robustness in optimization}

To control the level of robustness and give the flexibility to the designer in order to choose the appropriate robust solution, in this Section, we propose an approach to manipulate the extent of robust solution. This can be done by constructing a function and map the robust measure of equation 3 onto it. The constructed function should follow the reasoning of having the robust solution; the more the best. This invokes a dimensionless positive decreasing convex function which can not be approximated by high-order polynomial nor the cubic spline. To construct such a function, we introduce an approximation method as follows, which 
was first used in literature for some other purposes. We call this function the tunable robust function (TRF) onward. TRF is approximated piecewisely by mapping the scaled (between 0 and 100) robust measure of equation 3 , onto a dimensionless positive decreasing function $(T R F(R)$ where $R$ is scaled).

To start, designer will propose a level of desired robustness $(L R)$. Then, TRF is constructed on four regions, namely $L R \pm d$. Of course $d$ is a free parameter set by the designer, which can be changed appropriately. To guarantee the convexity of the TRF ${ }^{a}$, suppose that the derivative of TRF has the following form:

$$
\begin{gathered}
\frac{d T R F}{d R}=A e^{\alpha(R)}, \\
A>0, \quad \alpha^{\prime \prime}(R)>0
\end{gathered}
$$

taking $\alpha$ as a smooth function of robust measure in equation 3 and having integrated equation 6 , we reach to

$$
A \Delta_{k} R \int_{0}^{1} e^{\alpha^{(k)}(\xi)} d \xi=\Delta_{k} T R F
$$

where $\Delta_{k} T R F=T R F\left(R_{k+1}\right)-T R F(R-k), \alpha^{(k)}=\alpha^{(k)}\left(\xi^{(k)}\right), \xi^{(k)}=\frac{R-R_{k}}{R_{k+1}-R_{k}}\left(R_{k} \leq R \leq R_{k+1}\right)$ and $k$ shows the region the TRF is approximated. Figure 1 pictures the situation clearly.

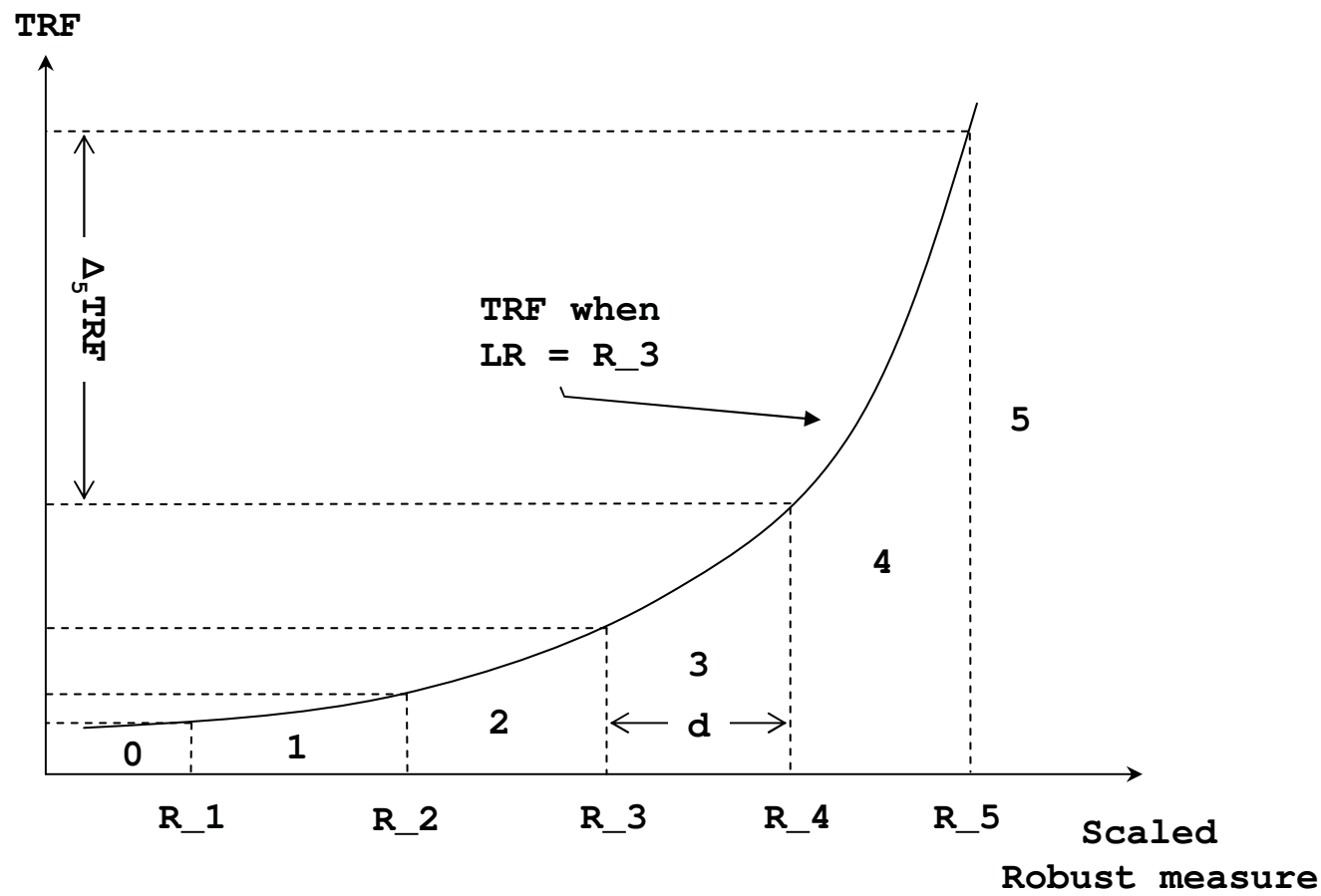

Figure 1: Piecewise Robust Functions (TRF)

Positivity and convexity bring about some conditions on $\alpha$ which reads as follows. In the given region, the lower value of the TRF on the current region is the same as the upper value of the afore region $\left(\alpha^{(k)}(0)=\right.$ $\left.\alpha^{(k-1)}(1)\right)$. This guarantees the continuity and the smoothness of the TRF, since the left and right value of the derivative has the same value. To satisfy this condition, a simple linear function

$$
\alpha^{(k)}(\xi)=a_{k} \xi+b_{k},
$$

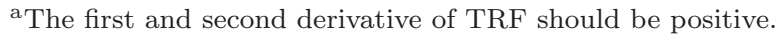


can be used if

$$
\begin{array}{r}
a_{k}=\frac{A \Delta_{k} R e^{b_{k}}\left(e^{a_{k}}-1\right)}{\Delta_{k} T R F}, \\
b_{k}=a_{k-1}+b_{k-1} .
\end{array}
$$

This gives a recurrent relation for calculation of $a_{k}$ and $b_{k}$. As the values of TRF at the boundaries of each region do not depend on the value of scaled robust function $(\mathrm{R})$, they are considered being fixed using:

$$
\begin{array}{r}
\Delta_{k} T R F=\beta n \Delta_{k-1} T R F, \\
\quad \beta>1, \quad \Delta_{0} T R F=1 .
\end{array}
$$

with $n$ denoting the number of objective functions. $\beta n$ guarantees to have a increasing exponential function in which the $k$ th region is desired to the $k+1$ th one in sense of minimization. As a final consideration, using 7 , if $k$ moves from 1 to 4 , the TRF can be approximated piecewisely:

$$
T R F^{(k)}=T R F_{k}+\Delta_{k} T R F \frac{e^{a_{k} \xi^{(k)}}-1}{e_{a_{k}}-1} \quad\left(R_{k} \leq R \leq R_{k+1}\right)
$$

where $T R F_{k}=T R F\left(R_{k}\right)$ and $R_{k}$ is the value of the scaled robust measure on the boundary of the region $k$ (Figure 1).

To have a function defined in $\mathbb{R}$, it is possible to choose a simple exponential function

$$
T R F^{(0)}=e^{A\left(R-R_{1}\right)}
$$

for the region of $R<R_{1}$. From this, it follows that

$$
a_{0}=0, \quad b_{0}=0,
$$

are the initial condition and hence $T R F^{(0)}$ changes to $T R F^{(0)}=1$.

Parameter A can be chosen as $\frac{1}{R_{5}-R_{1}}$ to have the same dimensionality as of $R^{-1}$.

As a comment, a positive solution of equation 11 always exists by taking

$$
a_{k}^{0}>1-A \Delta_{k} R e^{b_{k}} / \Delta_{k} T
$$

as an initial condition. This can be shown mathematically as follows.

First, we note the Banach fixed point theorem. The theorem says that any contraction map ${ }^{\mathrm{b}}$ has a unique fixed point ${ }^{\mathrm{c}}$.

Lets take the following map, where $x=a_{k}$ and $w=A \Delta_{k} R e^{b_{k}} / \Delta_{k} T$

$$
g(x)=\ln (1+x / w) .
$$

If we find the fixed point of $g(x)(g(x)=x)$, then it means that we are able to find the solution for the equation 11, that is $x=a_{k}$. To do so, following the Banach fixed point theorem, it is required that the above map $g(x)$ be a contractive map. To realize this, from calculus, we know that for any differentiable function g,

\footnotetext{
${ }^{\mathrm{b}} \mathrm{A}$ function $\mathrm{g}: \mathrm{X} \mapsto \mathrm{X}$ from a metric space $(\mathrm{X}, \mathrm{d})$ to itself is said to be a contraction if there is a constant $C \in(0,1)$ such that

for all $x, y \in \mathrm{X}$.

$$
d(g(x), g(y)) \leq C . d(x, y)
$$

${ }^{\mathrm{c}} \mathrm{A}$ point $a \in \mathrm{X}$ is said to be a fixed point of a function $\mathrm{g}: \mathrm{X} \mapsto \mathrm{X}$ if and only if $\mathrm{g}(\mathrm{a})=\mathrm{a}$.
} 


$$
g(x)-g(y)=g^{\prime}(t)(x-y)
$$

for some $t$ between $x$ and $y$. Therefore, we have

$$
\ln (1+x / w)-\ln (1+y / w)=\frac{1}{w+t} \cdot(x-y) .
$$

Thus,

$$
|\ln (1+x / w)-\ln (1+y / w)|=\left|\frac{1}{w+t}\right||x-y| .
$$

Since, $\frac{1}{w+t}$ is the constant term $(C)$ in Banach fixed point theorem, it should be between 0 and 1 . Therefore, $\frac{1}{w+t}<1$ and hence $x>1-w$ provides a contractive map. This can be taken as the initial condition for the iteration procedure for convergence to $x=a_{k}$.

It should be noted that with this method, the designer is able to cover all the robustness ranges, while the robust measure is scaled between 0 to 100. In the extreme points of 100 and 0 , the most robust solution (100 percent) and the regular (optimum solution) are generated. Obviously, one may expect the overlap between the optimum solution and the robust one under some cases.

Since the robust measure of equation 3 is introduced as a minimum objective function, we use the increasing TRF (the minimum is better).

Compared with the existing methods, the proposed approach is efficient in finding the robust design. This derives from the fact that the existing methods exploit the variance as the robust measure for which a huge number of points for each searching point (design) is needed to calculate the variability. This is a exhaustive and meanwhile not efficient approach for the expensive functions in terms of time and calculation. Furthermore, it is to be noted that the proposed method does not require a presumed probability distribution of variation and it only adds one simple objective function to the problem regardless of the dimension of the problem.

\section{Engineering test problem: Welded beam design}

In this section, we put into test the new approach in order to find the robust design for welded beam. The Pareto and robust frontiers are generated using the Directed Search Domain (DSD) method. ${ }^{16}$ The DSD method is able to generate an evenly distributed Pareto set in a general formulation, which is based on shrinking a search domain in a selected area on the Pareto frontier.

In this optimization problem, ${ }^{18}$ a beam is to be welded in two sides to a large case tolerating the force of $\mathrm{P}=(6000,54.8,54.8) \mathrm{lb}$ (Figure 2). The welding is to be done for minimum cost $\left(F_{1}\right)$ to design the beam for the minimum deflection $\left(F_{2}\right)$. The shear stress in weld $(\tau)$, bending stress in the beam $(\sigma)$, buckling load on the bar $(\mathrm{P})$ and end deflection of the beam $(\delta)$ are the design constraints adopted from. ${ }^{18}$ Taking thickness of the beam (b), width of the beam (t), length of the welded joint (l) and thickness of the weld (h) as the design variables and $\mathrm{L}=(14,3.5,3.5)$ inch as the uncertain fuzzy parameter, the best possible configuration is a favorite under the following formulation:

$$
\begin{aligned}
\text { Minimise } & F_{1}=1.105 h^{2} l+0.048 t b(14+l), \\
\text { Minimise } & F_{2}=\text { Deflection }=\delta=\frac{4 P L^{3}}{E t^{3} b}, \\
\text { s.t. } & \tau \leq 13600 \\
& \sigma \leq 30000 \\
& h \leq b \\
& P c \geq P \\
& 0.125 \leq b \leq 5, \quad 0.1 \leq t \leq 10, \\
& 0.1 \leq l \leq 10, \quad 0.125 \leq h \leq 5,
\end{aligned}
$$




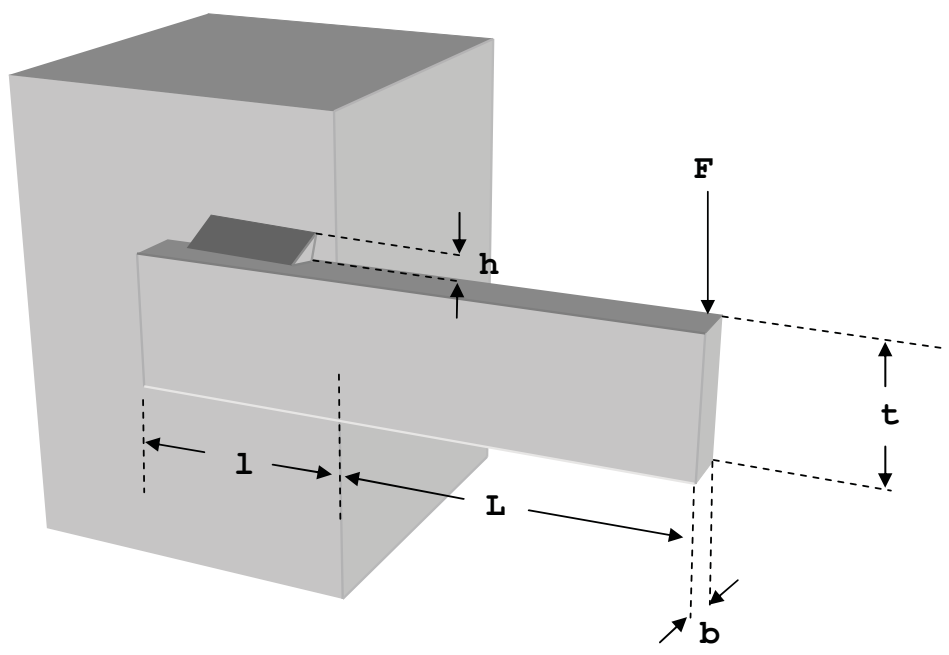

Figure 2: welded beam design

where

$$
\begin{aligned}
& \sigma=\frac{6 P L}{t^{2} b}, \\
& P c=64746.02(1-0.3 t) t b^{3} \text {, } \\
& \tau=\sqrt{\tau^{\prime 2}+\tau^{\prime \prime 2}+l \tau^{\prime} \frac{\tau^{\prime \prime}}{\sqrt{0.25\left(l^{2}+(h+t)^{2}\right)}}}, \\
& \tau^{\prime \prime}=P(14+0.5 l) \frac{\sqrt{0.25\left(l^{2}+(h+t)^{2}\right)}}{2\left(0.71 h l\left(l^{2} / 12+0.25(h+t)^{2}\right)\right)}, \\
& \tau^{\prime}=\frac{P}{\sqrt{2} h l}, \\
& P=(6000,54.8,54.8) l b, L=(14,3.5,3.5) i n, E=30 e 6 p s i .
\end{aligned}
$$

Using the DSD algorithm the global frontier is drawn. To find the robust solutions, the TRF method is used and a 50 and 100 percent frontiers are generated. As discussed before, all the robust solutions lie between the non-dominated frontier and 100 percent robust one. This three generated solutions provide useful information to the designer. As can be seen, the less sensitive part of Pareto frontier indicates the best solution as not only it is optimum but shows less variation in presence of the noise and uncertainty. A designer would choose these solutions provided the enough resources. It can be seen that using the TRF and the fuzzy variance of 500 and 2 for $\mathrm{P}$ and L, respectively, an appropriate design can be made. Furthermore, it is obvious that choosing the cost between 12 to 16 pounds does not bring any significant change to the optimal deflection and 100 percent robust deflection. This shows that, incurring the cost in the given range, the designer can design a structure which is optimal and also the utmost in robustness.

From other perspective, as can be seen in figure, the solution values degrade more when the robustness increases from 50 percent to 100 percent than it does from 0 percent to 50 percent. This shows that the optimality and robustness does not act linearly contrast to each other. Hence, the designer should stick to the robustness range of 50 percent as the designs are giving better results, which are closer to optimal.

For the matter of comparison between optimal and robust design, Figure, 4 is produced.

This figure shows the design of the beam corresponding to one of the $100 \%$ robust and optimal solutions 


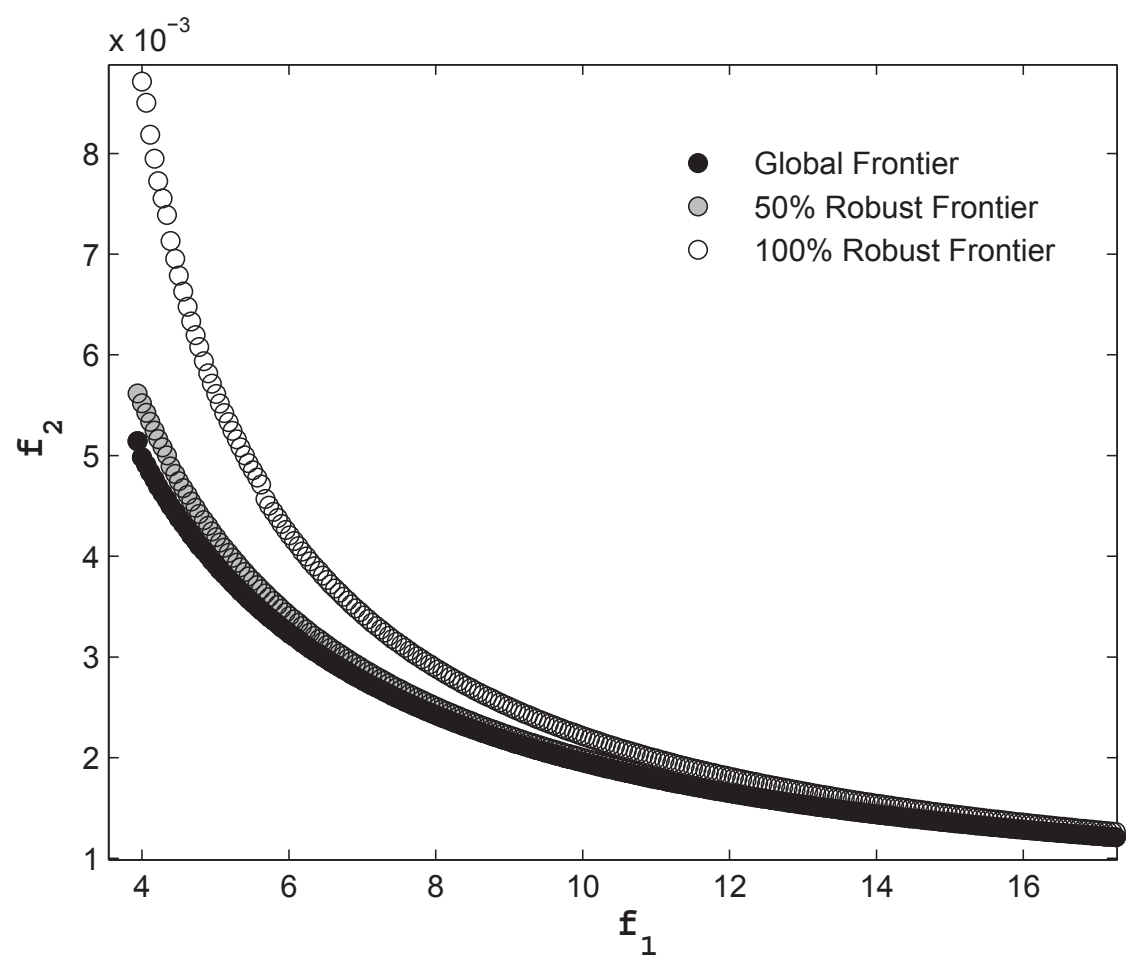

Figure 3: Design of Welded beam
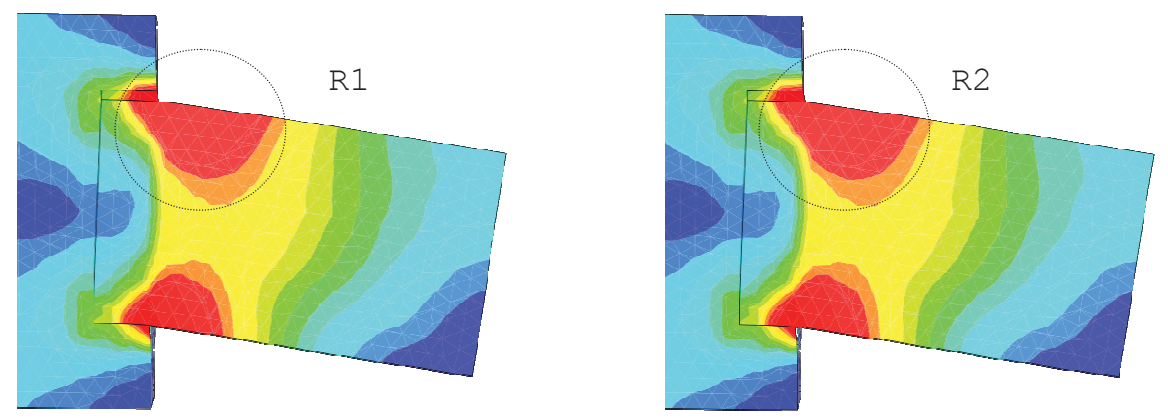

Robust designs under $27 \mathrm{kN}$ (left) and $32 \mathrm{kN}$ (right)
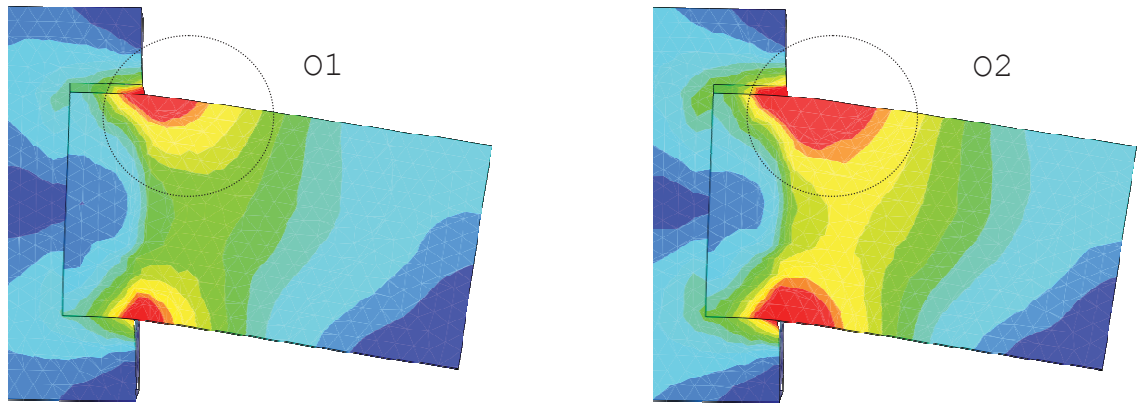

Optimal design under $27 \mathrm{kN}$ (left) and $32 \mathrm{kN}$ (right)

Figure 4: Comparison of the robust and optimal design of a beam 
taken from optimisation procedure (solutions of which is depicted in Figure 3). The meaning of robust design is illustrated perfectly in this Figure. As can be seen, the stress in robust beam did not change significantly by changing the force from 27 to $32 \mathrm{kN}$. Meanwhile, in the optimal beam design, stress changed under more pressure of $32 \mathrm{kN}$. To confirm our afore explanations, looking at Figure 4, one may note that the optomal design gives the better solution than the robust one, though the robust design introduces a stable configuration.

\section{Conclusion}

This paper introduced a flexible robust design methodology which not only is able to guarantee the robust design solutions but also provide the desired level of robustness set by the DM as the requirements in uncertain environment. Although any kinds of uncertainty can be handled, we implement the fuzzy value uncertainty on the parameters which is realized to be the most proper one. Using the analysis of tolerance and the mean and variance of fuzzy parameters, the transmitted variation into the constraints is calculated and the uncertain formulation is converted to the deterministic one. To realize the robust design, a robust measure is developed and based on it a tunable robust function (TRF) is constructed piecewisely. The TRF maps the proposed robust measure into a dimensionless positive convex function which its shape depends on the level of robustness the DM demands. The proposed approach is tested on an engineering problem and the results are promising. It is shown that we are able to set an upper bound of robust frontier using the proposed approach. All the other robust solutions, with any level of robustness, lies between the introduced frontiers.

\section{Acknowledgments}

The authors would like to thank the school of MACE at the University of Manchester for the financial support. The help and support of the administrative and technical staff of the University of Manchester are also greatly acknowledged.

\section{References}

\footnotetext{
${ }^{1}$ Bryne, D., "Taguchi approach to parameter design," Quality Progress, 1987, pp. 19-26.

${ }^{2}$ Taguchi, G., Elsayed, E., and Hsiang, T., Quality engineering in production systems, McGraw-Hill Companies, 1989.

${ }^{3}$ Ross, P., Taguchi techniques for quality engineering: loss function, orthogonal experiments, parameter and tolerance design, McGraw-Hill Professional, 1995.

${ }^{4}$ Ramakrishnan, B. and Rao, S., "A robust optimization approach using Taguchi's Loss Function for solving nonlinear optimization problems," Advances in Design Automation, 1991: Presented at the 1991 ASME Design Technical Conferences17th Design Automation Conference, September 22-25, 1991, Miami, Florida, American Society of Mechanical Engineers, 1991, p. 241.

${ }^{5}$ Sundaresan, S., Ishii, K., and Houser, D., "A procedure using manufacturing variance to design gears with minimum transmission error," Journal of Mechanical Design, Vol. 113, 1991, pp. 318.

${ }^{6}$ Mohandas, S., "Multiobjective optimization dealing with uncertainty," Advances in Design Automation, 1989: Design optimization: presented at The 1989 ASME design technical conferences-15th design automation conference, Montreal, Quebec, Canada, September 17-21, 1989, Amer Society of Mechanical, 1989, p. 241.

${ }^{7}$ Box, G. and Fung, C., "Studies in quality improvement: minimizing transmitted variation by parameter design," Center for Quality and Productivity Improvement. Report No. 8. University of Wisconsin-Madison, 1986.

${ }^{8}$ Rao, S., Optimization theory and applications, 1983.

${ }^{9}$ Parkinson, A., Sorensen, C., and Pourhassan, N., "A general approach for robust optimal design," Journal of Mechanical Design, Vol. 115, 1993, pp. 74 .

${ }^{10}$ Cox, N., "How to perform statistical tolerance analysis," NASA STI/Recon Technical Report N, Vol. 87, 1987, pp. 24582.

${ }^{11} \mathrm{Su}, \mathrm{J}$. and Renaud, J., "Automatic differentiation in robust optimization," AIAA Journal-American Institute of Aeronautics and Astronautics, Vol. 35, No. 6, 1997, pp. 1072-1079.

${ }^{12}$ Messac, A. and Ismail Yahaya, A., "Multiobjective robust design using physical programming," Structural and Multidisciplinary Optimization, Vol. 23, No. 5, 2002, pp. 357-371.

${ }^{13}$ Messac, A., "Physical programming: Effective optimization for computational design," AIAA journal, Vol. 34, No. 1, 1996, pp. 149-158.

${ }^{14}$ Deb, K. and Gupta, H., "Searching for robust Pareto-optimal solutions in multi-objective optimization," Proceedings of the Third Evolutionary Multi-Criteria Optimization (EMO-05) Conference (Also Lecture Notes on Computer Science 3410), Springer, 2005, pp. 150-164.

${ }^{15}$ Chen, W., Allen, J. K., Tsui, K.-L., and Mistree, F., "A Procedure For Robust Design: Minimizing Variations Caused By Noise Factors And Control Factors," ASME Journal of Mechanical Design, Vol. 118, 1996, pp. 478-485.
} 
${ }^{16}$ Erfani, T. and Utyuzhnikov, S. V., "Directed Search Domain: A Method for Even Generation of Pareto Frontier in Multiobjective Optimisation," Engineering Optimization, 2010, pp. DOI 10.1080/0305215X.2010.497185.

${ }^{17}$ Erfani, T. and Utyuzhnikov, S. V., "Handling Uncertainty and Finding Robust Pareto Frontier in Multiobjective Optimization Using Fuzzy Set Theory," 51st AIAA/ASME/ASCE/AHS/ASC Structures, Structural Dynamics, and Materials Conference et al, Orlando, Florida, 2010.

${ }^{18}$ Rao, S., Engineering optimization: theory and practice, Wiley-Interscience, 1996. 